\title{
Design of Robust Dynamic Inversion Control Laws using Multi-Objective Optimization
}

\author{
Gertjan Looye*and Hans-Dieter Joos ${ }^{\dagger}$ \\ German Aerospace Center \\ DLR-Oberpfaffenhofen \\ Institute for Robotics and Mechatronics \\ D-82234 Wessling, Germany \\ E-mails: \{gertjan.looye,dieter.joos\}@dlr.de
}

May 5, 2001

\begin{abstract}
The design of robust attitude control laws for a civil aircraft with nonlinear dynamic inversion and multiobjective optimization is discussed. Dynamic inversion is a methodology that achieves linearization and decoupled command responses of the closedloop system via inverse model equations in the feedback loop. Using a linear outer loop controller the desired dynamic behavior is imposed. For tuning the free controller parameters, multi-objective optimization is used. The required robustness is achieved via a multi-model approach, as well as local robustness measures (e.g. gain and phase margins) as optimization criteria. As a new approach, not only the linear controller gains are optimized, but also physical parameters in the inverse model that are considered uncertain in the design model. The resulting control laws are used as inner loops of an autoland system, which was assessed for JAR-AWO requirements and successfully flight tested.
\end{abstract}

\footnotetext{
* Research Engineer, PhD candidate

${ }^{\dagger}$ Research Engineer, PhD

Copyright (C)2001 by the German Aerospace Center DLR (Deutsches Zentrum für Luft- und Raumfahrt e.V.). Published by the American Institute of Aeronautics and Astronautics, Inc., with permission.
}

\section{Introduction}

Dynamic Inversion (DI) is a straight forward methodology for designing multi-variable control laws for nonlinear systems. DI has been used in numerous aerospace applications, especially for controllers that operate in highly nonlinear flight regimes, such as post-stall ${ }^{4}, 1$. A DI controller consists of two parts. In an inner loop, nonlinear inputoutput behavior of the plant is canceled by feedback control laws, consisting of inverse nonlinear model equations. The closed loop system is reduced to a set of integrators. In an outer loop, a linear controller is used to impose desired command response behavior. The control laws perform uniformly over the flight envelope covered by the inverted model equations, so that gain scheduling is avoided.

Unfortunately, Dynamic Inversion control laws may show very poor robustness to uncertainties in the design model. This problem is usually coped with by trying to recover the required robustness level in the design of the linear loops 4,1 .

We will use a different approach, based on best possible tuning of free synthesis parameters in the linear, as well as the nonlinear control laws. The synthesis parameters in the nonlinear control laws are physical parameters in the inverse model equations that are 
considered uncertain in the design model. Parameter synthesis is performed using multi-objective optimization. Robustness to parametric uncertainties is addressed via a multi-model approach ${ }^{7}$. Unspecified uncertainties are accounted for by using local robustness measures, such as gain and phase margins, as optimization criteria.

In this paper we will discuss the design of attitude control laws for a civil aircraft. These form the inner loops for an autoland system that was developed for two aircraft (RealCAM and ATTAS) in a project called REAL (Robust and Efficient Autoland control Laws design $)^{9,2}$. The results in this paper are based on the ATTAS design. ATTAS (Advanced Technologies Testing Aircraft System) is a small passenger aircraft with two jet engines, operated by DLR as in-flight simulator as well as fly-by-wire testbed. One of the main objectives of REAL was to develop an efficient design process resulting in robust autoland control laws. The process proposed by DLR is described in Ref. ${ }^{9}$. The design work presented in this paper is performed along the same lines, but the emphasis will be on methodology aspects.

The paper is structured as follows. In section 2 the aircraft model and its implementation in Modelica is discussed. In section 3 the DI control laws are described and in section 4 implementation aspects are discussed. In section 5 parameter synthesis via multi-objective optimization is presented. Finally, assessment results and conclusions can be found in sections 6 and 7 .

\section{The aircraft model}

As a modeling platform, the object-oriented modeling software Modelica/Dymola ${ }^{3}$ is used, in which a flight-mechanics class library ${ }^{11}$ is available. The aircraft model is depicted in Fig. 1. The model components are implemented one-to-one as software objects. The central object in Figure 1, body contains the Newton-Euler equations of motion ${ }^{13}$. The aerodynamics are in aeroRef and engine models in engRef1,2. The object motiATTAS contains rate and position limited linear actuator models for ailerons, elevator, and rudder. The object airport contains airport terrain, and ILS models. The lower-right

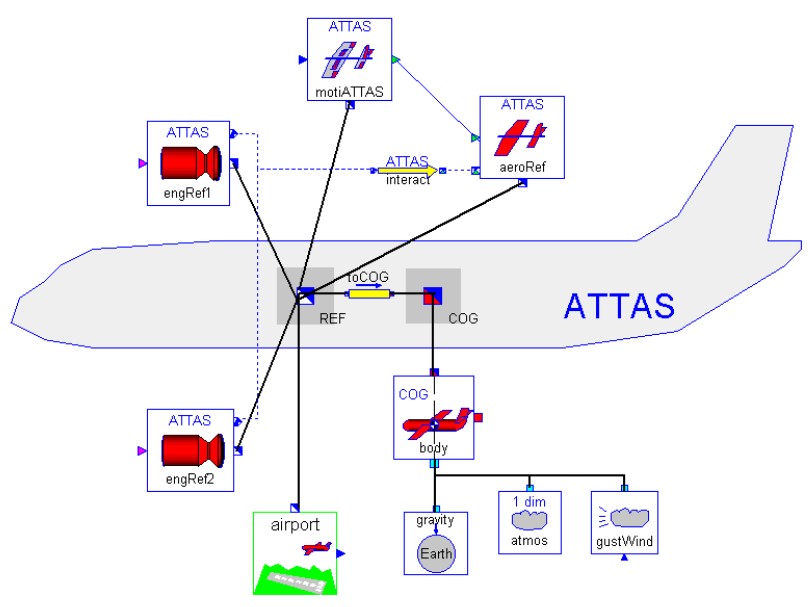

Figure 1: Aircraft model in Dymola

blocks are gravity, atmospheric and wind models. The underlying modeling language, Modelica ${ }^{10}$ is equation-based. The depicted interconnections represent kinematic constraints and energy flows.

From the model implemented in Modelica, Dymola sorts and solves the model equations according to defined inputs and outputs and generates simulation code for the selected engineering environment (in this case Matlab/Simulink ${ }^{T M}$ ). The model outputs are the measurements available to the control system: calibrated airspeed $V_{\text {cas }}$, true airspeed $V_{\text {tas }}$, ground speed $V_{\text {ground }}$, body angular rates $p, q, r$, attitude angles $\phi, \theta, \psi$, load factors $n_{x}, n_{y}, n_{z}$ flight path angles $\chi, \gamma$, angle of attack $\alpha$, vertical speed $V_{Z}$, deviations from the ILS beam $\epsilon_{L O C}, \epsilon_{G L D}$, radio and barometric altitude $H_{\text {ra }}, H_{\text {baro }}$, the mean fan shaft speed of both engines $N_{1}$, the aircraft mass $m$, and the center of gravity location $x_{C O G}$. The available controls are defined as inputs: ailerons $\delta_{A}$, elevator $\delta_{E}$, rudder $\delta_{R}$, and throttle settings of the two engines $\delta_{T 1,2}$. The stabilizer is used for initial trimming of the aircraft. In addition, there are white noise inputs for turbulence and ILS noise models. A list of parameters is defined that allows to set mass and loading conditions, wind, etc. prior to simulation. Also tolerances on aerodynamic coefficients ${ }^{13}$ and inertial parameters are included, such as $\Delta C_{Y}$, 
$\Delta C_{l_{0}}, \Delta C_{n_{0}}, \Delta C_{l_{p}}, \Delta C_{l_{r}}, \Delta C_{n_{p}}, \Delta C_{n_{r}}$, etc. These are relative tolerances, e.g.:

$$
C_{l_{0}}=C_{l_{0_{n} \circ m}}\left(1+\Delta C_{l_{0}}\right)
$$

where $C_{l_{0_{\text {nom }}}}$ is the nominal parameter value. The tolerances vary from $10 \%$ (longitudinal coefficients) to $30 \%$ (lateral and ground effect-related coefficients).

\section{Dynamic Inversion Control Laws}

The designed control laws serve as inner loops for an autoland system ${ }^{9}$. The control objective is to robustly track pitch attitude commands $\theta_{c}$, roll commands $\Phi_{c}$, and heading rate commands $\dot{\Psi}_{c}$. The pitch commands are provided by the glide slope and flare modes. The localizer mode provides coordinated roll angle and heading rate commands. The align mode provides roll commands to keep the aircraft on the localizer beam, and heading angle commands to align the aircraft with the runway centre line in case of cross-wind (de-crab). In the align mode the $\psi$-loop is closed via $\dot{\psi}_{c}$ and a gain $K_{\psi}$. In tuning the DI controller parameters, both cases (heading loop open and closed) will therefore be considered.

The controller structure is depicted in Figure 2. The meaning and design of the blocks will be explained in the course of this section.

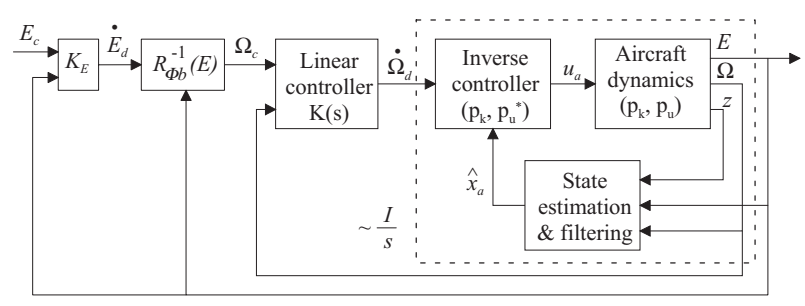

Figure 2: Attitude control system

In the outer loop, attitude rate demands $\dot{E}_{d}$ are generated using:

$$
\dot{E}_{d}=K_{E}\left(E_{c}-E\right)
$$

where $E=[\phi, \theta, \psi]^{T}$ and $K_{E}$ is a diagonal gain matrix (for simplicity of notation, the $\psi$-loop is considered closed), and $E_{c}$ is the commanded aircraft attitude. From $\dot{E}_{d}$ angular rate commands $\Omega_{c}$ are computed using the inverse transformation:

$$
\Omega_{c}=R_{\phi b}^{-1}(E) \dot{E}_{d}
$$

where

$$
R_{\Phi b}=\left[\begin{array}{ccc}
1 & \sin \phi \tan \theta & \cos \phi \tan \theta \\
0 & \cos \phi & -\sin \phi \\
0 & \sin \phi / \cos \theta & \cos \phi / \cos \theta
\end{array}\right]
$$

and $\Omega=[p, q, r]^{T}$

We design tracking control laws for $\Omega_{c}$ using Dynamic Inversion 12,4 . To this end, $\Omega$ as a model output is differentiated once. This results in the aircraft moment equation of motion (see e.g. ref. $^{13}$ ):

$$
\begin{aligned}
\dot{\Omega} & =I\left(p_{k}, p_{u}\right)^{-1}\left[M_{A}\left(x_{a}, \dot{x}_{a}, p_{k}, p_{u}\right)\right. \\
& +M_{u}\left(x_{a}, p_{k}, p_{u}\right) u_{a}+M_{T}\left(x_{a}, \delta_{T 1,2}, p_{k}, p_{u}\right) \\
& \left.-\Omega \times I\left(p_{k}, p_{u}\right) \Omega\right]
\end{aligned}
$$

where $M_{A}$ and $M_{T}$ denote moments due to aerdynamic and thrust forces respectively. The moments due to aerodynamic control deflections $u_{a}=$ $\left[\begin{array}{lll}\delta_{A}, & \delta_{E}, & \delta_{R}\end{array}\right]^{T}$ have been separated from $M_{A}$ as $M_{u}\left(x_{a}, p_{k}, p_{u}\right)$. The (airmass referenced) state vector is $x_{a}$. The terms of eq. 4 depend on defined model parameters (section 2). Those have been divided into on-line known parameters $\left(p_{k}\right)$ and uncertain parameters $\left(p_{u}\right)$.

Since the three control inputs in $u_{a}$ are primarily moment generating devices around the three aircraft body axes, $M_{u}\left(x_{a}, p_{k}, p_{u}\right)$ is (in the normal flight regime) non-singular. By inversion of eq. 4 we obtain the following Dynamic Inversion control law:

$$
\begin{aligned}
u_{a} & =M_{u}\left(\hat{x}_{a}, p_{k}, \hat{p}_{u}\right)^{-1}\left[I\left(p_{k}, \hat{p}_{u}\right) \dot{\Omega}_{d}\right. \\
& -M_{A}\left(\hat{x}_{a}, \hat{\dot{x}}_{a}, p_{k}, \hat{p}_{u}\right)-M_{T}\left(\hat{x}_{a}, \delta_{T 1,2}, p_{k}, \hat{p}_{u}\right) \\
& \left.+\Omega \times I\left(p_{k}, \hat{p}_{u}\right) \Omega\right]
\end{aligned}
$$

where $\dot{\Omega}_{d}$ is the new control input, representing angular rate demands (see Figure 2). Note that we replaced $x_{a}$ with $\hat{x}_{a}$ and $p_{u}$ with $\hat{p}_{u}$. In the inverse control laws, the states are not obtained from 
internal integration, but from on-line measurement and/or estimation from sensor outputs $z$ (listed in section 2). The parameters contained in $p_{k}, p_{u}$ also appear in the inverse model equations. The known parameters can be adapted on-line. The uncertain parameters are for example relative uncertainties in aerodynamic coefficients and in the inertia tensor (section 2). In a simulation model, uncertain parameters can be set freely for analysis purposes, but in the inverse model equations their values are fixed (vector $\hat{p}_{u}$ ). In the ideal case, i.e. when $\hat{p}_{u}=p_{u}$ and $\hat{x}_{a}=x_{a}$ ), substitution of eq. 5 into eq. 4 gives:

$$
\dot{\Omega}=\dot{\Omega}_{d} \Longleftrightarrow \Omega=\frac{1}{s} \dot{\Omega}_{d}
$$

Note that the input-output behaviour is de-coupled and reduces to an integrator (Figure 2). It is common practice to use nominal values for the parameters in $\hat{p}_{u}$, which corresponds to inverting the nominal aircraft model. We found, that selecting offnominal values in $\hat{p}_{u}$ may considerably increase robustness of the DI controller to variations in parameters contained in $p_{u}$. The selection is done using optimization, which will be discussed in section 5 . Finally, as a linear control law $\mathrm{K}(\mathrm{s})$ we use (Figure 2):

$$
\dot{\Omega}_{d}=K_{i} \frac{1}{s}\left(\Omega_{c}-\Omega\right)+K_{p} \Omega
$$

The integrator on $\left(\Omega_{c}-\Omega\right)$ is required to compensate for static errors in the moment equations. The optimization of the linear gains (including $K_{E}$ ) will be discussed in section 5 .

\section{Implementation aspects}

Dynamic Inversion control laws provide full control deflections consisting of a quasi-steady component that trims the aircraft, and a varying component due to manoeuvering. Because of model deviations, the quasi-steady part may have an offset. As a consequence, the closed loop system may show considerable transient behavior when the controller is initialized. For this reason, the following algorithm is used:

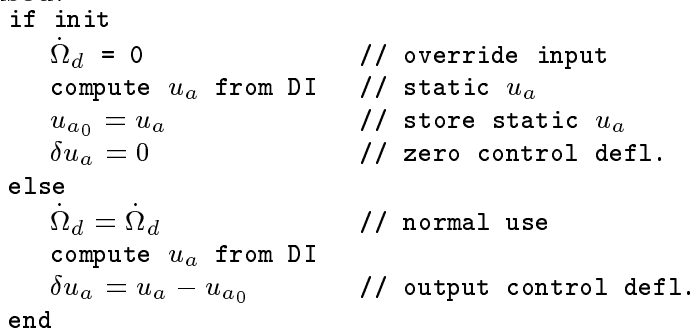

By setting $\dot{\Omega}_{d}=0$ at the initial computation, only the current 'trimmed' deflections are obtained. These are subtracted from the control inputs from then onwards, so that the controller only provides control deflection "delta's" with respect to the control deflections at initialization.

The DI control laws require full state information from the aircraft dynamics. The airmass referenced velocities along the body axes $\left(V_{A}=\right.$ $\left.\left[u_{a_{b}}, v_{a_{b}}, w_{a_{b}}\right]^{T}\right)$ are computed from the true airspeed $V_{\text {tas }}$, the angle of attack $\alpha$ and the side slip angle $\beta$ using the relations:

$$
\begin{aligned}
& u_{a_{b}}=V_{\text {tas }} \sqrt{\frac{1-\sin ^{2} \beta}{1+\tan ^{2} \alpha}} \\
& v_{a_{b}}=V_{t a s} \sin \beta \\
& w_{a_{b}}=u_{a_{b}} \tan \alpha
\end{aligned}
$$

The side slip angle is not measured and therefore has to be estimated. Writing the side force equation in the aerodynamic model in the form:

$$
C_{Y}=C_{Y_{\beta}}(M, \alpha, \ldots) \beta+C_{Y_{\text {rest }}}\left(\delta_{R}, M, \alpha, \ldots\right)
$$

Using:

$$
n_{y}=\frac{\frac{1}{2} \rho_{0} V_{c a s}^{2} S C_{Y}}{m g}
$$

the side slip angle can be estimated from:

$$
\beta_{\text {est }}=\frac{1}{C_{Y_{\beta}}}\left[\frac{n_{y} m g}{1 / 2 \rho_{0} V_{\text {cas }}^{2} S}-C_{Y_{\text {rest }}}\right]
$$

The right hand term can be computed using the coefficients from the model and the available measurements. To reduce the propagation of noise due to turbulence, the signal is complementarily filtered with:

$$
\dot{\beta}_{i}=\frac{-n_{y} g+g \sin \phi \cos \theta}{V_{\text {tas }}}-r \cos \alpha+p \sin \alpha
$$


During localizer tracking, the DI controller has to track co-ordinated roll and heading rate commands. For this reason, simulation 1 is performed, from which criteria such as rise time and overshoot of a combined roll and heading rate step command are computed. During the align phase, roll and heading angles are controlled independently. The roll angle is used to track the localizer, the heading angle is used for decrab in case of cross-wind. Therefore, simulation 2 is performed, The criterion psst is meant as a measure for the settling time, although a different measure is used to actually compute the criterion. The $\dot{\psi}$-command is computed from additional feedback of $\psi$ :

$$
\dot{\psi}_{c}=k_{\psi}\left(\psi_{c}-\psi\right)
$$

from which criteria are computed based on a step command on $\psi_{c}$.

Disturbance rejection is assessed in simulation 3 through a heavy cross wind step.

The design of the complete autoland controller ${ }^{9}$ was based on JAR-AWO criteria ${ }^{5}$. For the inner loops, no explicit criteria were available and therefore had to be formulated. Table 1 lists the criteria that were actually used for the final design.

\section{$\underline{5.2}$ Robustness}

The aircraft model depends on a set of parameters, that contains e.g. the mass, center of gravity location and uncertainties on aerodynamic coefficients. Robustness to variations in these parameters, as well as unspecified model uncertainty is achieved in three ways.

1. Parameters $p_{k}$ that can be determined on-line, can directly be fed to the controller, The DI controller automatically adapts to these parameters. In this case, the aircraft mass and center of gravity location are known.

2. Robustness to parametric uncertainties is achieved via a multi-model approach. A set of model cases with worst-case parameter combinations (w.r.t. selected criteria from Table 1) is searched and added to the nominal model in a multi-model set. The criteria in table 1 are computed for all model cases. The complete set of criteria is addressed in the optimization, allowing for trade-off between criteria under nominal and worst-case conditions.

3. Robustness to uncertainties other than described by tolerances on parameters (e.g. time delays, unmodeled dynamics) is addressed via local unspecific robustness margins as optimization criteria. To this end, minimum gain and phase margins are specified.

In Table 1 the unspecific robustness criteria, i.e. gain and phase margins at different actuator and sensor locations in the control loop, as well as the minimum damping, are listed. Note that the latter also incorporates the requirement of stability of the internal (zero) dynamics ${ }^{12}$.

\subsection{Scaling of criteria}

The criteria are scaled, keeping in mind that a value less than one is considered satisfactory. The applied scalings are given in Table 2. Scaling can be per-

\begin{tabular}{|c|c|c|c|c|}
\hline $\begin{array}{l}\text { crit, } \\
\text { unit }\end{array}$ & $\begin{array}{l}\text { bad } \\
\text { low }\end{array}$ & $\begin{array}{c}\text { good } \\
\text { low }\end{array}$ & $\begin{array}{c}\text { demand } \\
\text { value }\end{array}$ & type \\
\hline phrt (s) & - & - & 2.5 & $\mathrm{c}$ \\
\hline phos $(-)$ & - & - & 0.05 & $\mathrm{c}$ \\
\hline phst $\left({ }^{\circ}\right)$ & - & - & 0.1 & $\mathrm{~m}$ \\
\hline psdd (s) & - & - & 10 & $\mathrm{~m}$ \\
\hline phds $(\% / \mathrm{s})$ & - & - & 0.1 & $\mathrm{~m}$ \\
\hline dda $(\% / \mathrm{s})$ & - & - & 20 & $\mathrm{~m}$ \\
\hline psrt (s) & - & - & 5 & $\mathrm{c}$ \\
\hline $\operatorname{psos}(-)$ & - & - & 0.05 & $\mathrm{c}$ \\
\hline $\operatorname{psst}\left({ }^{\circ}\right)$ & - & - & 0.2 & $\mathrm{~m}$ \\
\hline pher $\left({ }^{\circ}\right)$ & - & - & 0.1 & $\mathrm{~m}$ \\
\hline $\mathrm{ddr}\left({ }^{\circ} / \mathrm{s}\right)$ & - & - & 10 & $\mathrm{~m}$ \\
\hline phwe $\left(^{\circ}\right)$ & - & - & 6.7 & $\mathrm{~m}$ \\
\hline drpeak $\left({ }^{\circ}\right)$ & - & - & 25 & $\mathrm{~m}$ \\
\hline $\operatorname{gmada}(\mathrm{dB})$ & 4 & 6 & 1 & $\mathrm{~m}$ \\
\hline $\operatorname{pmada}\left({ }^{\circ}\right)$ & 30 & 60 & 1 & $\mathrm{c}$ \\
\hline $\operatorname{gmadr}(\mathrm{dB})$ & 4 & 6 & 1 & $\mathrm{~m}$ \\
\hline $\operatorname{pmadr}\left({ }^{\circ}\right)$ & 30 & 60 & 1 & c \\
\hline gmsp (dB) & 4 & 6 & 1 & $\mathrm{~m}$ \\
\hline $\operatorname{pmsp}\left({ }^{\circ}\right)$ & 30 & 60 & 1 & $\mathrm{c}$ \\
\hline gmsr $(\mathrm{dB})$ & 4 & 6 & 1 & $\mathrm{~m}$ \\
\hline $\operatorname{pmsr}\left({ }^{\circ}\right)$ & 30 & 60 & 1 & $\mathrm{c}$ \\
\hline damp (-) & 0.6 & 0.7 & 1 & $\mathrm{c}$ \\
\hline $\begin{array}{l}\text { Legend: } \\
\mathrm{m}=\text { minir }\end{array}$ & & & & 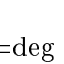 \\
\hline
\end{tabular}

Table 2: Scaling of criteria

formed by division of the criterion by its demand 
value (criterion phrt ... drpeak):

$$
\hat{c}_{i}(T)=c_{i}(T) / d_{i}
$$

where $c_{i}(T)$ and $d_{i}$ are the computed value and demanded value of criterion $i$ respectively, and $T$ denotes the current set of tuning parameters. Scaling can also be done using so-called 'good-bad' values ${ }^{6}$, (see Ref. ${ }^{9}$ for an example). Here, a special type of 'good-bad'scaling is used, as will be explained for gmada in Figure 4. The demand is that the gain

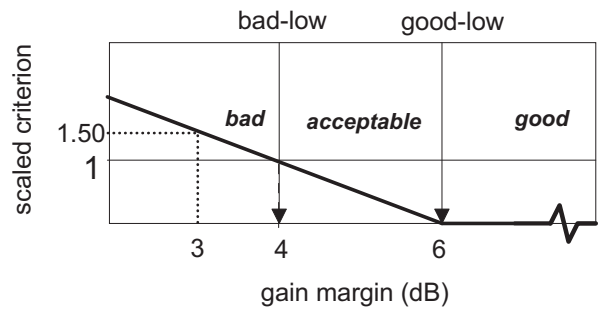

Figure 4: Scaling of gmada with good-bad values

margin is at least $4 \mathrm{~dB}$ ('bad-low'). Any value larger than 6 ('good-low') is considered equally good and therefore scaled to 0 . Below $6 \mathrm{~dB}$, the scaling increases linearly, such that a value of 1 is reached for the bad-low value of $4 \mathrm{~dB}$. Any value between 4 and $6 \mathrm{~dB}$ is acceptable, any value lower than $4 \mathrm{~dB}$ is considered bad. As an example, if the gain margin is 3 $\mathrm{dB}$, its scaled value equals 1.5 .

Some of the criteria in Table 2 are treated as inequality constraint. For example, a rise time of $2.5 \mathrm{~s}$ is demanded. If this is satisfied, there is no point to further minimize this criterion, since this will unnecessarily go at the cost of stability and control effort.

\subsection{Parameter synthesis}

For parameter synthesis, the multi-objective optimization tool MOPS (Multi-Objective Parameter Synthesis $^{6}$ ) is used. As a first step, only the gains of the lateral part of the linear controller (eq. 6) are tuned. The resulting values can be found in the first column of Table 3 .

Using this gain set, a worst-case analysis was performed. It was assumed that worst-cases occur in the corners of the parameter space. For this reason,

\begin{tabular}{|l|c|c|c|l|}
\hline $\begin{array}{l}\text { tuning } \\
\text { par. }\end{array}$ & $\begin{array}{c}\text { (1) nom. } \\
\text { design }\end{array}$ & $\begin{array}{c}\text { (2) m.mod. } \\
\text { design 1 }\end{array}$ & $\begin{array}{c}\text { (3) m.mod. } \\
\text { design 2 }\end{array}$ & unit \\
\hline \hline \multicolumn{5}{|l|}{ linear controller gains: } \\
\hline$K_{i_{p}}$ & 2.9 & 2.28 & 2.52 & - \\
$K_{p_{p}}$ & 3.47 & 2.86 & 2.92 & $s^{-1}$ \\
$K_{i_{r}}$ & 2.0 & 2.56 & 2.17 & - \\
$K_{p_{r}}$ & 1.6 & 1.95 & 1.96 & $s^{-1}$ \\
$K_{\phi}$ & 0.41 & 0.41 & 0.41 & $s^{-1}$ \\
$K_{\psi}$ & 0.70 & 0.70 & 0.70 & $s^{-1}$ \\
\hline aerodynamic coefficients in $\hat{p}_{u}$ (eq. 5) \\
\hline$\Delta C_{Y}$ & 0 & 0 & -0.3 & - \\
$\Delta C_{l_{0}}$ & 0 & 0 & -0.3 & - \\
$\Delta C_{n_{0}}$ & 0 & 0 & -0.23 & - \\
$\Delta C_{l_{p}}$ & 0 & 0 & -0.07 & - \\
$\Delta C_{l_{r}}$ & 0 & 0 & -0.3 & - \\
$\Delta C_{n_{p}}$ & 0 & 0 & $-0,3$ & - \\
$\Delta C_{n_{r}}$ & 0 & 0 & -0.15 & - \\
$\Delta C_{n_{\delta}}$ & 0 & 0 & 0.3 & - \\
\hline In Figure $2:$ & \multicolumn{5}{|l}{} \\
$K_{E}=\operatorname{diag}\left(\left[K_{\Phi}, K_{\theta}, K_{\psi}\right]\right)$ \\
$K_{i}=\operatorname{diag}\left(\left[K_{i_{p}}, K_{i_{q}}, K_{i_{r}}\right]\right)$ \\
$K_{p}=\operatorname{diag}\left(\left[K_{p_{p}}, K_{p_{q}}, K_{p_{r}}\right]\right)$ \\
\hline
\end{tabular}

Table 3: Controller synthesis parameters

the uncertain parameters (tolerances on lateral aerodynamic coefficients and moments of inertia) were set to their minimum or maximum values and the criteria in Table 1 were evaluated for all parameter combinations. Three models were selected: the one with maximum (scaled) value for damp (worst damping), the one with maximum gmsr (lowest gain margin at yaw rate sensor, unstable), and the one with maximum pmadr (minimum phase margin at rudder actuator). These criteria were most sensitive to the parameter variations and considered most critical.

The three worst model cases were added to the nominal model in a multi-model set. For this set of model cases, a new parameter synthesis was performed. First, only the linear gains were optimized. The values are given in the second column of Table 2. The results were not satisfactory, as will be discussed in section 6 . In the off-nominal model cases, the scaling of some criteria was slightly relieved compared with Table 2.

During the third parameter synthesis, not only the linear gains, but also eight of fourteen aerodynamic tolerances in the parameter vector $\hat{p}_{u}$ in the DI con- 

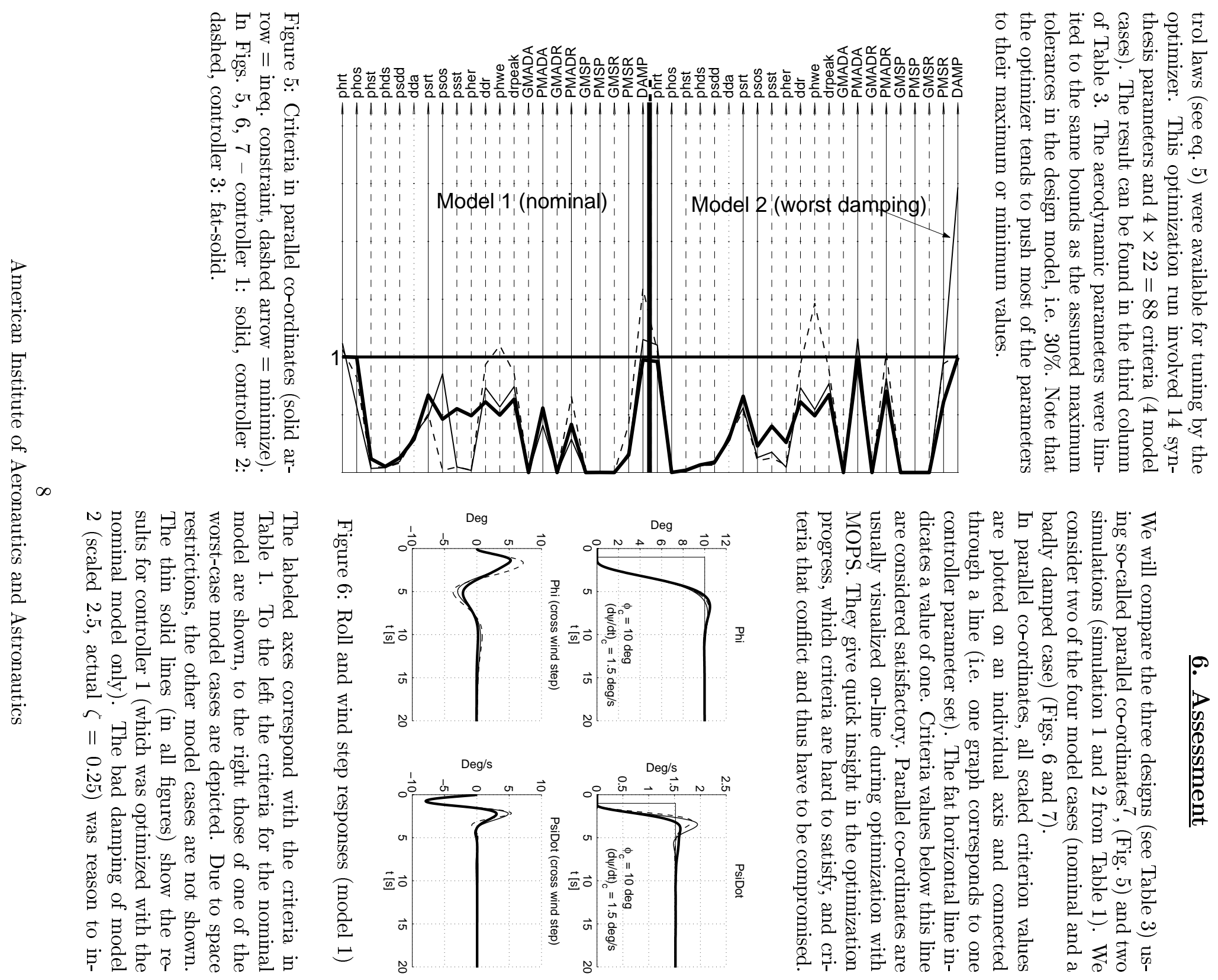

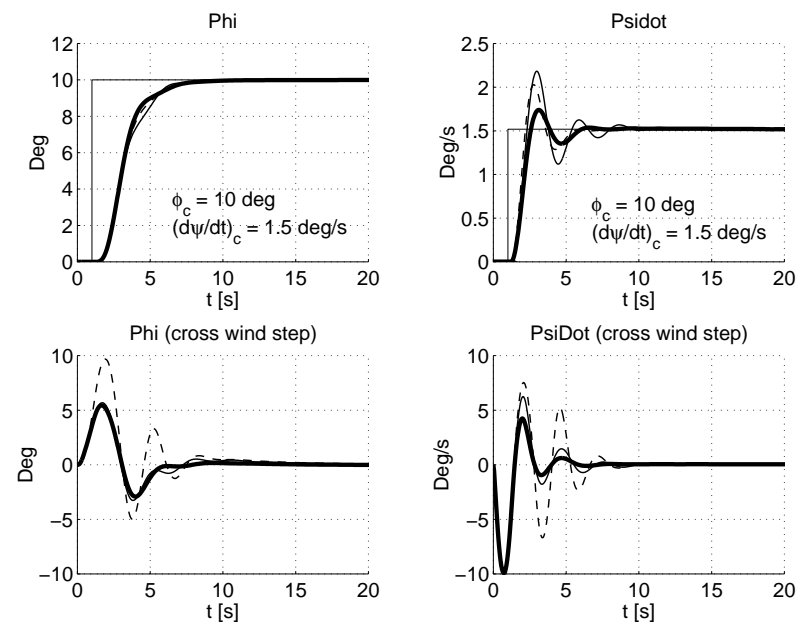

Figure 7: Roll and wind step responses (model 2)

clude this model in the multi-model set. The results from optimization of the linear controller parameters only, with the selected multi-model set, are represented using the dashed line. The damping is increased to a satisfactory level (criterion below 1-line in Fig. 5), but this goes clearly at the cost of rudder activity ( $d d r, d r p e a k)$, the roll response due to a side wind step phwe (see also Fig. 7), the phase margin at the rudder actuator pmadr, and the damping of the nominal model. The even worse damping in Fig. 7 (below) seems in contradiction with the better damping level, but inspection of the results revealed that the rudder actuator rate saturates due to its higher activity. In one of the model cases (not shown) this even causes instability. The fat solid line shows the results for controller 3 , for which linear gains, as well as model parameters $\in \hat{p}_{u}$ in the DI controller were optimized. From the parallel coordinates it is immediately clear, that all criteria are satisfied (this also holds for the two model cases not shown). Especially Fig. 7 shows considerable improvement.

Of course, with the gain set of controller 3 , a new parameter study was performed. All stability related criteria are satisfied for the investigated parameter combinations (no figure). In a number of cases the roll angle error $15 \mathrm{~s}$ after the roll command step (phst) and the roll angle response due to the wind step (phwe) degrade, but in no case instability due to rate saturation occurs.

\section{Conclusions}

The design of the inner loops of an autoland control using dynamic inversion and multi-objective optimization was discussed. The controller is robust to parametric uncertainties, such as tolerances in aerodynamic coefficients. This was achieved via a multimodel approach, and robustness indicators as criteria in multi-objective optimization. As inner loops in the autoland system ${ }^{9}$, the control laws were successfully flight tested in the DLR Advanced Technologies Testing Aircraft System (ATTAS) ${ }^{2}$.

The DI control laws explicitly depend on the same set of parameters as the design model. The parameters in the DI controller are set to their nominal values, except for those that can be determined on-line (mass, center of gravity location), and a number of tolerances on aerodynamic parameters. Those were used as additional synthesis parameters in the optimization, to achieve a good compromise in performance and stability between nominal and worst-case model cases.

The design was performed within the scope of the project REAL (Robust and Efficient Autoland control Law design) ${ }^{2}$. The tight schedule left little time for further in depth research. However, the results presented in this paper are encouraging. For example, the use of the structured singular value instead of loop-by-loop stability margins is considered as optimization criterion. For the search of worst model cases, advanced analysis methods are considered.

\section{Acknowledgment}

The ATTAS model implementation in Dymola/Modelica (and the underlying libraries) were made available by Dieter Moormann from the same institute as the authors. 


\section{$\underline{\text { References }}$}

[1] Richard J. Adams and Siva S. Banda. Robust Flight Control Design Using Dynamic Inversion and Structured Singular Value Synthesis. IEEE Transactions on Control Systems Technology, 1(2):80-92, June 1993.

[2] M. Bauschat, W. Mönnich, D. Willemsen, and G. Looye. Flight testing Robust Autoland Control Laws. In Proceedings of the AIAA Guidance, Navigation and Control Conference 2001, Montreal CA, 2001.

[3] H. Elmqvist. Object-oriented modeling and automatic formula manipulation in Dymola. In Scandinavian Simulation Society SIMS, Kongsberg, Norway, 1993.

[4] Dale Enns, Dan Bugajski, Russ Hendrick, and Gunter Stein. Dynamic Inversion: An Evolving Methodology for Flight Control Design. In AGARD Conference Proceedings 560: Active Control Technology: Applications and Lessons Learned, pages 7-1 - 7-12, Turin, Italy, May 1994. NATO-AGARD.

[5] Joint Aviation Authorities Committee. Joint Aviation Requirements, All Weather Operations. Technical report, JAAC, 1996.

[6] H.-D. Joos. A methodology for multi-objective design assessment and flight control synthesis tuning. Aerospace Science and Technology, 3(3):161-176, April 1999.

[7] G. Kreisselmeier and R. Steinhauser. Systematische Auslegung von Reglern durch Optimierung eines vektoriellen Gütekriteriums. Regelungstechnik, Heft 3, pages 76-79, 1979.

[8] A.A. Lambregts. Aircraft Automatic Control Systems Design, 1996. Lecture notes on 5 seminars given at Delft University of Technology, The Netherlands.
[9] Gertjan Looye, Hans-Dieter Joos, and Dehlia Willemsen. Application of an Optimisationbased Design Process for Robust Autoland Control Laws. In Proceedings of the AIAA Guidance, Navigation and Control Conference 2001, Montreal CA, 2001.

[10] S.E. Mattsson, M. Otter, and H. Elmqvist. Modelica Hybrid Modeling and Efficient Simulation. In Proceedings of the of the 38th IEEE Conference on Decision and Control, $C D C$ '99, December 1999. Also available from http://www.modelica.org.

[11] D. Moormnann, P.J. Mosterman, and G. Looye. Object-oriented computational model building of aircraft flight dynamics and systems. Aerospace Science and Technology, 3(3), April 1999.

[12] Jean Jacques E Slotine and Weiping Li. Applied Nonlinear Control. Prentice Hall, Englewood Cliffs, N.J., 1991.

[13] Brian L. Stevens and Frank L. Lewis. Aircraft Control and Simulation. Wiley-Interscience Publication. John Wiley \& Sons, Inc., New York, 1992. 